Agns Desolneux Lionel Moisan

Jean-Michel Morel

\title{
From Gestalt Theory to Image Analysis
}

A Probabilistic Approach

\section{4: Springer}




\section{Contents}

Preface ...........................................................................................................

1 1tleoduetim ....................................................................................................

1.1 Gestalt Theory and Computer Vision ......................................... 1

1.2 Basic Principles of Computer Vision ……………....................... 3

2 Gestalt Theory ….......................................................................................... 11

2.1 Before Gestaltism: Optic-Geometric Illusions ………...................... 11

2.2 Grouping Laws and Gestalt Principles ....................................... 13

2.2.1 Gestalt Basic Grouping Principles …………………........ 13

2.2.2 Collaboration of Grouping Laws ...................................... 17

2.2.3 Global Gestalt Principles ................................................... 19

2.3 Conflicts of Partial Gestalts and the Masking Phenomenon ............ 21

2.3.1 Conflicts ………........................................................ 21

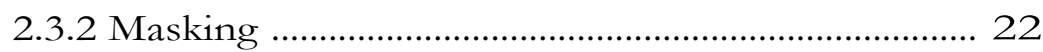

2.4 Quantitative Aspects of Gestalt Theory ........................................ 25

2.4.1 Quantitative Aspects of the Masking Phenomenon ............ 25

2.4.2 Shannon Theory and the Discrete Nature of Images ......... 27

2.5 Bibliographie Notes ................................................................ 29

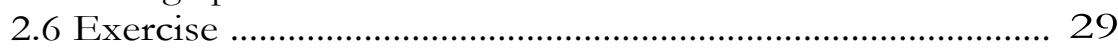

2.6.1 Gestalt Essay ……………………………………......... 29

3 The Helmholtz Principle ................................................................................ 31

3.1 Introducing the Helmholtz Principle: Three Elementary

Examples ………............................................................. 31

3.1.1 A Black Square an a White Background ........................... 31

3.1.2 Birthdays in a Class and the Role of Expectation ................ 34

3.1.3 Visible and Invisible Alignments ...................................... 36

3.2 The Helmholtz Principle and E-Meaningful Events ....................... 37

3.2.1 A First Illustration: Playing Roulette with Dostoievski ....... 39

3.2.2 A First Application: Dot Alignments .................................. 41

3.2.3 The Number of Tests .................................................... 42 
3.3 Bibliographic Notes $\quad \ldots 43$

3.4 Exercise ...................................................................... 44

3.4.1 Birthdays in a Class $\quad \ldots 44$

4 Estimating the Binomial Tail ............................................................... 47

4.1 Estimates of the Binomial Tail ................................................ 47

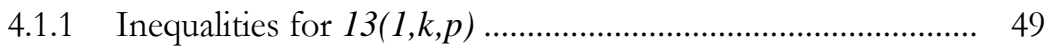

4.1.2 Asymptotic Theorems for $B(1, k, p)=\mathrm{P}[\mathrm{Si}>k] \ldots \ldots \ldots \ldots \ldots \ldots . . . .50$

4.1.3 A Brief Comparison of Estimates for $B(1, k, p)$................... 50

4.2 Bibliographic Notes ........................................................ 52

4.3 Exercises ..................................................................... 52

4.3.1 The Binomial Law ........................................................ 52

4.3.2 Hoeffding's Inequality for a Sum of Random Variables ...... 53

4.3.3 A Second Hoeffding Inequality ................................... 55

4.3.4 Generating Function ................................................. 56

4.3.5 Large Deviations Estimate ....................................... 57

4.3.6 The Central Limit Theorem ........................................... 60

4.3.7 The Tail of the Gaussian Law ........................................ 63

$5 \quad$ Alignments in Digital Images ...................................................... 65

5.1 Definition of Meaningful Segments ..................................... 65

5.1.1 The Discrete Nature of Applied Geometry ..................... 66

5.1.2 The A Contrario Noise Image ................................... 67

5.1.3 Meaningful Segments ............................................... 70

5.1.4 Detectability Weights and Underlying Principles .............. 72

5.2 Number of False Alarms ....................................................... 74

5.2.1 Definition ............................................................. 74

5.2.2 Properties of the Number of False Alarms ........................ 75

5.3 Orders of Magnitudes and Asymptotic Estimates ....................... 76

5.3.1 Sufficient Condition of Meaningfulness .......................... 77

5.3.2 Asymptotics for the Meaningfulness Threshold $k(l)$........... 78

5.3.3 Lower Bound for the Meaningfulness Threshold $k(l) \ldots \ldots \ldots . \quad 80$

5.4 Properties of Meaningful Segments ........................................ 81

5.4.1 Continuous Extension of the Binomial Tail ...................... 81

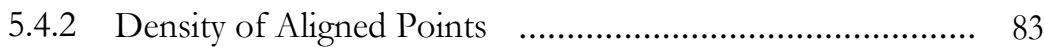

5.5 About the Precision $p$................................................................ 86

5.6 Bibliographic Notes ....................................................... 87

5.7 Exercises ...................................................................... 91

5.7.1 Elementary Properties of the Number of False Alarms ...... 91

5.7.2 A Continuous Extension of the Binomial Law ................ 91

5.7.3 A Necessary Condition of Meaningfulness ...................... 92 
6 Maximal Meaningfulness and the Exclusion Principle ........................ 95

6.1 Introduction …………………........................................... 95

6.2 The Exclusion Principle ………………………….................... 97

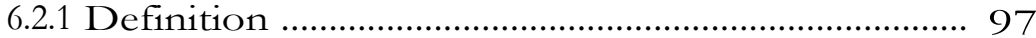

6.2.2 Application of the Exclusion Principle to Alignments ......... 98

6.3 Maximal Meaningful Segments ....................................................... 100

6.3.1 A Conjecture About Maximality ………………………...... 102

6.3.2 A Simpler Conjecture ....................................................... 103

6.3.3 Proof of Conjecture 1 Under Conjecture 2 ........................ 105

6.3.4 Partial Results About Conjecture 2 ……………….............. 106

6.4 Experimental Results ................................................................ 109

6.5 Bibliographical Notes .............................................................. 112

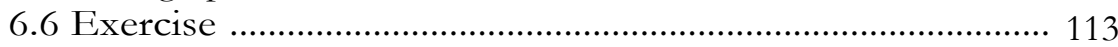

6.6.1 Straight Contour Completion …………………………..... 113

7 Modes of a Histogram ....................................................................................... 115

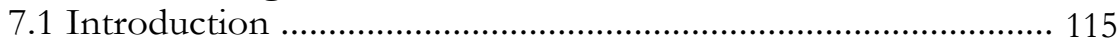

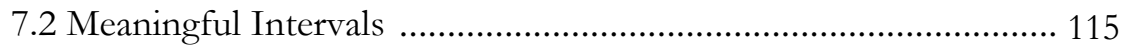

7.3 Maximal Meaningful Intervals .................................................... 119

7.4 Meaningful Gaps and Modes ...................................................... 122

7.5 Structure Properties of Meaningful Intervals ............................... 123

7.5.1 Mean Value of an Interval ............................................... 123

7.5.2 Structure of Maximal Meaningful Intervals ......................... 124

7.5.3 The Reference Interval ................................................... 126

7.6 Applications and Experimental Results …………....................... 127

7.7 Bibliographie Notes …………………………….................... 129

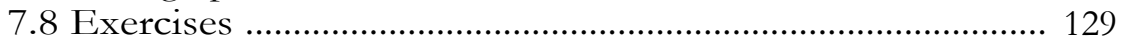

7.8.1 Kullback-Leibler Distance ................................................. 129

7.8.2 A Qualitative a Contrario Hypothesis .................................. 130

8 Vanishing Points ................................................................................................. 133

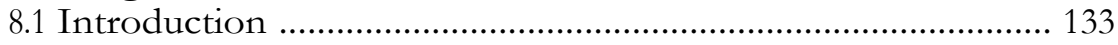

8.2 Detection of Vanishing Points .................................................... 133

8.2.1 Meaningful Vanishing Regions ......................................... 134

8.2.2 Probability of a Line Meeting a Vanishing Region .............. 135

8.2.3 Partition of the Image Plane into Vanishing Regions ........... 137

8.2.4 Final Remarks ............................................................... 141

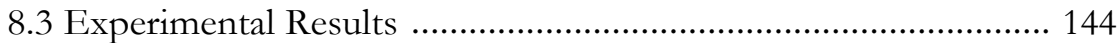

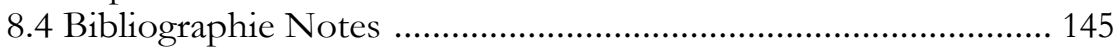

8.5 Exercises ............................................................................ 150

8.5.1 Poincar6-Invariant Measure an the Set of Lines .................. 150

8.5.2 Perimeter of a Convex Set .............................................. 150

8.5.3 Crofton's Formula ............................................................ 150 
9 Contrasted Boundaries ........................................................................ 153

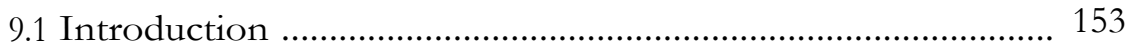

9.2 Level Lines and the Color Constancy Principle ……...................... 153

9.3 A Contrario Definition of Contrasted Boundaries ........................... 159

9.3.1 Meaningful Boundaries and Edges ………....................... 159

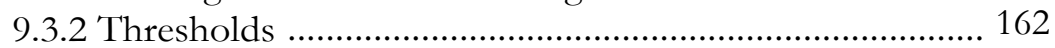

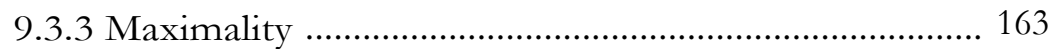

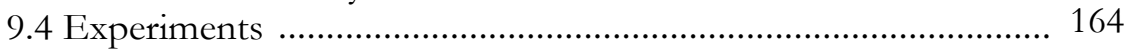

9.5 Twelve Objections and Questions ……………............................. 168

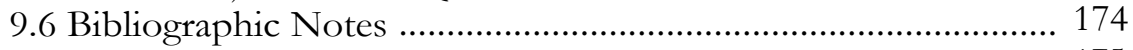

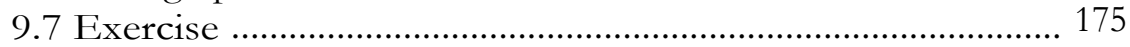

9.7.1 The Bilinear Interpolation of an Image .............................. 175

10 Variational or Meaningful Boundaries ? 177

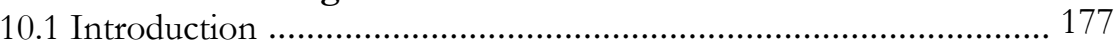

10.2 The "Snakes" Models ............................................................... 177

10.3 Choice of the Contrast Function $g$................................................... 180

10.4 Snakes Versus Meaningful Boundaries ……………..................... 185

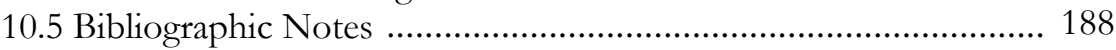

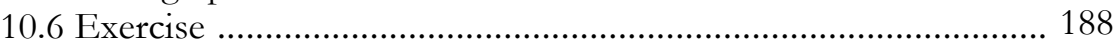

10.6.1 Numerical Scheme ......................................................... 188

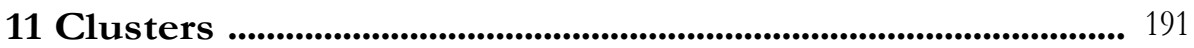

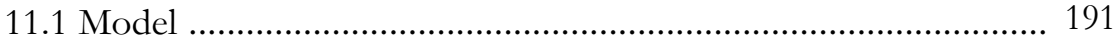

11.1.1 Low-Resolution Curves …………………….................. 191

11.1.2 Meaningful Clusters ......................................................... 193

11.1.3 Meaningful Isolated Clusters ……………………............. 193

11.2 Finding the Clusters ................................................................. 194

11.2.1 Spanning Tree ….............................................................. 194

11.2.2 Construction of a Curve Enclosing a Given Cluster ............ 194

11.2.3 Maximal Clusters ......................................................... 196

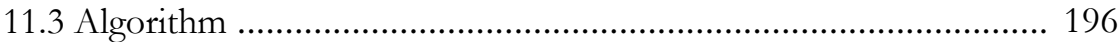

11.3.1 Computation of the Minimal Spanning Tree ....................... 196

11.3.2 Detection of Meaningful Isolated Clusters ......................... 197

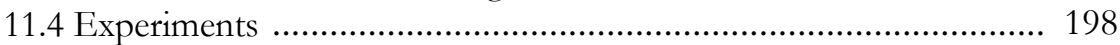

11.4.1 Hand-Made Examples ....................................................... 198

11.4.2 Experiment an a Real Image ............................................ 198

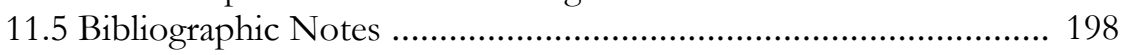

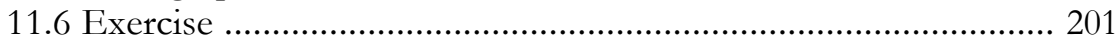

11.6.1 Poisson Point Process ........................................................ 201

12 Binocular Grouping …........................................................................... 203

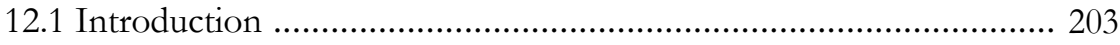

12.2 Epipolar Geometry …............................................................. 204

12.2.1 The Epipolar Constraint .................................................... 204

12.2.2 The Seven-Point Algorithm ................................................. 204 


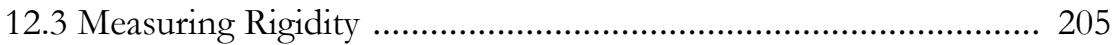

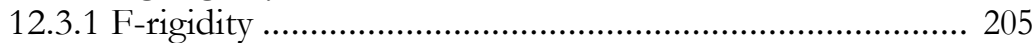

12.3.2 A Computational Definition of Rigidity ............................. 206

12.4 Meaningful Rigid Sets ............................................................. 207

12.4.1 The Ideal Case (Checking Rigidity) ……………….......... 207

12.4.2 The Case of Outliers ...................................................... 208

12.4.3 The Case of Nonmatched Points ....................................... 210

12.4.4 A Few Remarks ........................................................ 214

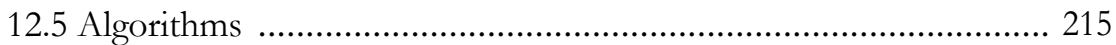

12.5.1 Combinatorial Search .................................................... 215

12.5.2 Random Sampling Algorithm ........................................... 216

12.5.3 Optimized Random Sampling Algorithm (ORSA) ……...... 217

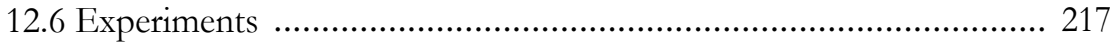

12.6.1 Checking All Matchings .................................................. 217

12.6.2 Detecting Outliers .............................................................. 219

12.6.3 Evaluation of the Optimized Random Sampling

Algorithm ………….................................................. 219

12.7 Bibliographie Notes ................................................................. 222

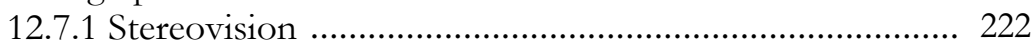

12.7.2 Estimating the Fundamental Matrix from Point Matches ..... 223

12.7.3 Robust Methods .......................................................... 224

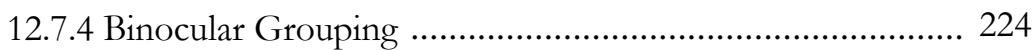

12.7.5 Applications of Binocular Grouping ............................... 225

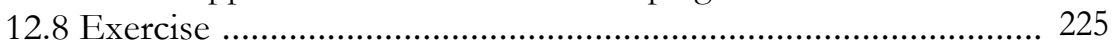

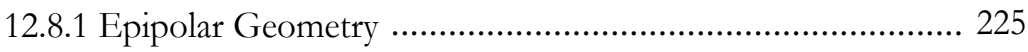

13 A Psychophysical Study of the Helmholtz Principle ............................. 227

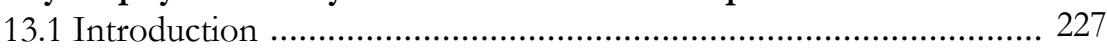

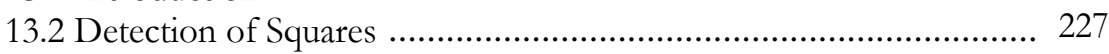

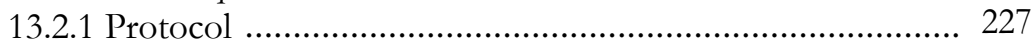

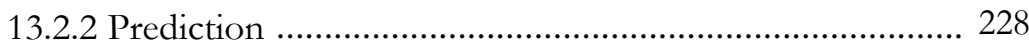

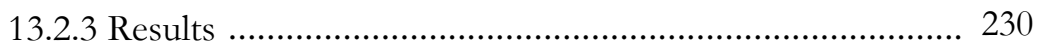

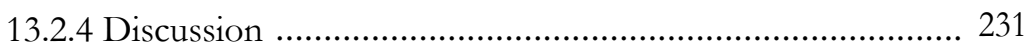

13.3 Detection of Alignments ............................................................ 231

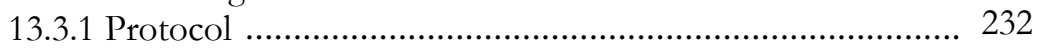

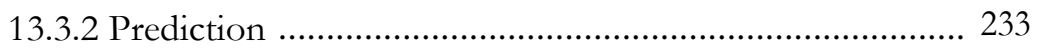

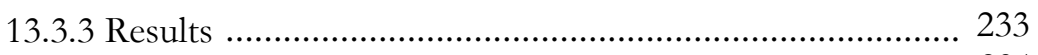

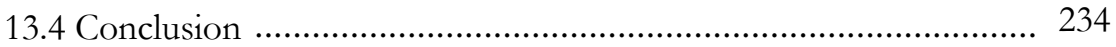

13.5 Bibliographie Notes ............................................................... 235

14 Back to the Gestalt Programme ................................................................... 237

14.1 Partial Gestalts Computed So Far ……………............................ 237

14.2 Study of an Example ……………………………………….... 240

14.3 The Limits of Every Partial Gestalt Detector ……......................... 242

14.3.1 Conflicts Between Gestalt Detectors .................................. 242 
14.3.2 Several Straight Lines or Several Circular Arcs? ............... 244

14.3.3 Influence of the A-contrario Model ................................ 246

14.4 Bibliographic Notes ........................................................ 247

15 Other Theories, Discussion ......................................................... 249

15.1 Lindenbaum's Theory ........................................................ 249

15.2 Compositional Model and Image Parsing ............................... 250

15.3 Statistical Framework ..................................................... 252

15.3.1 Hypothesis Testing ................................................... 252

15.3.2 Various False Alarms or Error Rates Compared to NFA ... 253

15.3.3 Comparison with Signal Detection Theory ....................... 254

15.4 Asymptotic Thresholds .......................................................... 255

15.5 Should Probability Be Maximized or Minimized? ..................... 256

References ............................................................................................................ 261

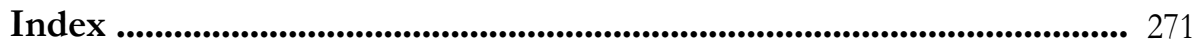

